\title{
Doenças crônicas, capacidade funcional, envolvimento social e satisfação em idosos comunitários: Estudo Fibra
}

\author{
Chronic diseases, functional ability, social involvement \\ and satisfaction in community-dwelling elderly: the Fibra study
}

Juliana Martins Pinto ${ }^{1}$

Anita Liberalesso Neri ${ }^{1}$

\footnotetext{
${ }^{1}$ Programa de Pós-

Graduação em

Gerontologia, Faculdade de Ciências Médicas,

Universidade Estadual de Campinas. R. Tessália

Vieira de Camargo 126, Cidade Universitária

Zeferino Vaz. 13.083-887

Campinas SP Brasil.

ju_fisio33@yahoo.com.br

${ }^{2}$ Departamento de

Psicologia Educacional,

Faculdade de Educação,

Universidade Estadual de
}

Abstract The scope of this article is to describe variations in the measurement of chronic diseases, functional ability, social involvement and satisfaction with respect to memory, problem solving, social relationships, environment, health services and transportation. This is done according to gender, age and income. It analyzes correlations between social involvement and functional ability in independent community dwelling-elderly aged 65 and above. 2,472 seniors without cognitive deficit, from probabilistic samples of seven Brazilian locations, were submitted to self-reported measurement concerning all variables, with the exception of grip strength and gait speed assessed by objective tests. Mean age was $72.2 \pm 5.5$ years and mean income was $3.9 \pm 4.9 \mathrm{MW} ; 65.7 \%$ were women, who had more diseases, worse functional performance and greater social involvement than men; those aged 80 and above and the poorest participants had worse functional performance and less social involvement. Correlations were observed between functional ability and social involvement. Level of income was related to satisfaction concerning memory, problem solving, health and transport services. Health, functionality and satisfaction interact in old age, influencing patterns of activity and social involvement.

Key words Muscle strength, Comorbidities, Social activities, Personal satisfaction, Old age
Resumo O objetivo deste artigo é descrever variações em medidas de doenças crônicas, capacidade funcional, envolvimento social e satisfação com relação à memória, capacidade de resolver problemas, relações sociais, ambiente, serviços de saúde e transportes, conforme gênero, idade e renda familiar, e analisar correlações entre envolvimento social e capacidade funcional, conforme gênero e idade, em idosos independentes, com 65 anos e mais, recrutados na comunidade. Foram submetidos 2.472 idosos sem déficit cognitivo, integrantes de amostras probabilísticas de sete localidades brasileiras a medidas de autorrelato sobre todas as variáveis, com exceção de força de preensão palmar e velocidade da marcha, avaliadas por testes objetivos. $A$ idade média foi 72,2 \pm 5,5 anos e a renda 3,9 \pm 4,9 SM; 65,7\% eram mulheres, que apresentaram mais doenças, pior desempenho funcional e maior envolvimento social do que os homens. Os de 80 anos ou mais e os mais pobres tiveram pior desempenho funcional e menor envolvimento social. Houve correlação entre capacidade funcional e envolvimento social. Renda relacionou-se com satisfação com memória, solução de problemas, serviços de saúde e transportes. Saúde e satisfação interagem na velhice, influenciando padrões de atividade $e$ de envolvimento social.

Palavras-chave Força muscular, Comorbidades, Atividades sociais, Satisfação pessoal, Velhice 


\section{Introdução}

Satisfação com a vida é o resultado da avaliação cognitiva que as pessoas fazem do grau de ajuste entre os recursos pessoais e sociais de que dispõem e suas necessidades e expectativas ${ }^{1,2}$. Nessa avaliação são considerados critérios pessoais e socioculturais. Juntamente com a satisfação com a vida e com afetos positivos e negativos, a satisfação referenciada a domínios integra o construto de bem-estar subjetivo ${ }^{3}$. As medidas de satisfação referenciada a domínios contribuem para esclarecer quais são os elementos que controlam as avaliações globais de satisfação com a vida e ajudam a ajustar o foco das intervenções profissionais e das políticas sociais e de saúde.

Os idosos costumam pontuar alto em avaliações de satisfação. Porém, na presença de doenças crônicas e de dependência funcional, os escores tendem a ser mais baixos, assim como na presença de dor crônica, inatividade, depressão e experiência de eventos incontroláveis, que são ocorrências mais comuns na velhice avançada do que na inicial ${ }^{4,5}$. No entanto, apenas quando esses eventos desafiam fortemente a adaptação, ocorre declínio na satisfação, e não simplesmente em função do avanço da idade ${ }^{6}$.

A funcionalidade e a incapacidade resultam da relação complexa entre condições de saúde e de competência comportamental e as circunstâncias ecológicas e sociais sob as quais o indivíduo vive ${ }^{7}$. A capacidade funcional atua indiretamente sobre a satisfação dos idosos, por meio de sua relação com a mobilidade, a funcionalidade, a frequência de atividades e o grau de envolvimento ativo ${ }^{8}$. A presença de três ou mais comorbidades relaciona-se com declínio funcional, depressão, uso de medicamentos, redução da participação social, pior qualidade de vida, altos custos dos serviços de saúde e altos níveis de mortalidade ${ }^{9}$. As mulheres, aqueles com mais de 80 anos e os que têm menor renda são mais afetados por essas condições do que os homens, os idosos mais jovens e os que têm maior renda ${ }^{10}$.

As condições de saúde e a funcionalidade física afetam o envolvimento dos idosos com as interações sociais, o desempenho de papéis sociais primários e a participação política. O desempenho de atividades físicas, cognitivas, sociais e organizacionais complexas ou avançadas permite aos idosos serem considerados ativos, produtivos e socialmente envolvidos. No entender de Reuben et al. ${ }^{11,12}$, as atividades avançadas de vida diária (AAVD) sobrepõem-se às atividades ins- trumentais de vida diária (AIVD) e às atividades básicas de vida diária (ABVD). A diminuição na frequência de AAVD pode ser sinal de limitações da mobilidade, do equilíbrio e da força muscular, de doenças crônicas e de fragilidade ${ }^{13}$, assim como do processo adaptativo de seletividade socioemocional ${ }^{14}$, que repercute na motivação para as atividades e para a interação social. Como a diminuição nas AAVD é precursora de limitações em AIVD e ABVD, a observação do envolvimento social é elemento de prevenção a ser cultivado nos vários níveis de atenção aos idosos ${ }^{15}$.

Condições de saúde e incapacidade funcional que comprometem o envolvimento social dos idosos contribuem para sua insatisfação com a vida ${ }^{16}$. Esta contribui para a restrição do idoso ao ambiente doméstico, o que diminui suas chances de usufruir das vantagens da interação social e associa-se ao aumento do risco para mortalidade, morbidade, incapacidade física e cognitiva, inatividade e depressão ${ }^{17}$.

A partir das contribuições pioneiras da teoria da atividade ${ }^{18}$ e do afastamento ${ }^{19}$, da pesquisa longitudinal de Rowe e Kahn sobre velhice bem-sucedida ${ }^{20} \mathrm{e}$ da intervenção política de âmbito mundial da Organização Mundial da Saúde em prol do envelhecimento ativo ${ }^{21}$, nos últimos cinquenta anos a atividade e o envolvimento social têm sido apontados como elementos fundamentais à manutenção da saúde, da cognição $\mathrm{e}$ da funcionalidade física. Não há estudos populacionais brasileiros a esse respeito. Dadas as peculiaridades das influências educacionais, socioeconômicas e culturais sobre o processo de envelhecimento no Brasil, é relevante estudar o envolvimento social e a satisfação em idosos, considerando-se condições de saúde física e capacidade funcional, gênero e idade de indivíduos de diferentes regiões do país.

\section{Objetivos}

Este estudo teve como objetivos descrever variações em medidas de doenças crônicas, capacidade funcional, envolvimento social e satisfação com relação aos domínios memória, capacidade de resolver problemas no dia-a-dia, relações sociais, ambiente, serviços de saúde e transportes, conforme as variáveis gênero, idade e renda familiar, assim como analisar correlações entre envolvimento social e capacidade funcional em idosos com 65 anos e mais, independentes, recrutados na comunidade. 


\section{Métodos}

Foram utilizados dados do banco eletrônico do Estudo Fibra (acrônimo de Fragilidade em Idosos Brasileiros), polo Unicamp ${ }^{22}$, cujo projeto foi aprovado pelo Comitê de Ética em Pesquisa com Seres Humanos da Faculdade de Ciências Médicas da Universidade Estadual de Campinas (CONEPE/ FCM/UNICAMP). Os dados foram coletados em sete localidades brasileiras com distintos níveis de desenvolvimento socioeconômico. Essas localidades foram escolhidas por conveniência. Em cada uma delas foi selecionada uma amostra probabilística de setores censitários, em cujos domicílios foram recrutados idosos de 65 anos e mais, compondo cotas de homens e mulheres de 65 a 69, 70 a 74, 75 a 79 e 80 anos e mais, cotas essas proporcionais ao seu tamanho na população de idosos de cada localidade segundo o IBGE ${ }^{23}$.

Foram adotados como critérios de inclusão: ter idade igual ou superior a 65 anos, compreender as instruções, concordar em participar e ser residente permanente no domicílio e no setor censitário. Com base no estudo de Fried et al. ${ }^{24}$, do qual foi derivada a definição operacional de fragilidade usada pelo Estudo Fibra, foram adotados como critérios de exclusão: presença de problemas de memória, atenção, orientação espacial e temporal e comunicação, sugestivos de déficit cognitivo; incapacidade permanente ou temporária para andar; perdas localizadas de força e afasia decorrentes de Acidente Vascular Encefálico (AVE); comprometimento grave da motricidade, da fala ou da afetividade associados à doença de Parkinson; déficits auditivo ou visual graves e doença em fase terminal.

Todos os idosos foram avaliados quanto a variáveis sociodemográficas, pressão arterial, antropométricas, de fragilidade e status cognitivo. O escore obtido por cada um deles no MiniExame do Estado Mental (MEEM) menos um desvio-padrão ${ }^{25}$ foi utilizado como nota de corte para participação num segundo conjunto de medidas que incluiu condições de saúde e funcionalidade e variáveis psicossociais. Entre elas estavam as variáveis de interesse para este estudo. Todos os participantes assinaram um termo de consentimento livre e esclarecido.

\section{Participantes}

A amostra deste estudo foi composta por 2.472 idosos sem déficit cognitivo sugestivo de demência, independentes, recrutados na comunidade, integrantes das amostras de Belém (PA)
(558), Parnaíba (PI) (284), Campina Grande (PB) (229), Poços de Caldas (MG) (299), Ermelino Matarazzo (SP) (291), Campinas (SP) (655) e Ivoti (RS) (156).

\section{Instrumentos e medidas}

Variáveis socioeconômicas. Idade, gênero e renda familiar. A avaliação foi feita por meio de três questões de autorrelato. As idades foram agrupadas em quatro faixas: 65-69; 70-74; 7579; $80 \mathrm{e}+$. Os valores de renda foram agrupados em cinco categorias de $\mathrm{SM}: \leq 1 ; 1,1$ a $3 ; 3,1$ a $5 ; 5,1$ a $10 ;>10$ SM.

Doenças crônicas. Perguntou-se aos idosos se, no ano anterior, algum médico havia dito que eram portadores de cardiopatias, hipertensão arterial, diabetes, doença cerebrovascular, tumor/ câncer, artrite, pneumopatias, depressão e osteoporose. O idoso respondia sim ou não para cada doença. As frequências foram agrupadas em três faixas: nenhuma, uma ou duas e três ou mais.

Capacidade funcional. Foram tomadas medidas de força de preensão manual e de velocidade da marcha. A medida de força de preensão palmar em quilogramas força $\left(\mathrm{Kg}_{\mathrm{f}}\right)$ foi obtida por meio de dinamômetro hidráulico, modelo Jamar (Lafayette Instruments ${ }^{\circledast}$, Lafayette, Indiana, Estados Unidos), que foi colocado na mão dominante de cada idoso. Em posição sentada, cada um realizou três tentativas intercaladas por um minuto de repouso. A prova de velocidade da marcha incluiu a tomada de três medidas consecutivas do tempo em segundos que cada idoso gastou para percorrer 4,6 m no plano, em linha reta, seguindo um traçado previamente demarcado com fita adesiva colorida. Foi permitido o uso de bengala ou andador ${ }^{26}$. Foram considerados com baixa força de preensão os idosos cuja média das três medidas em $\mathrm{Kg}_{\mathrm{f}}$ caiu abaixo do $1^{\mathrm{o}}$ quintil da amostra, com ajustamento por sexo e índice de massa corporal $\left(\mathrm{IMC}=\right.$ peso/altura $\left.{ }^{2}\right)$. Foram considerados lentos aqueles cuja média do tempo de marcha, em segundos, foi superior ao percentil 80 da amostra, com ajuste por sexo e altura ${ }^{24}$.

Foram calculadas notas de corte especificas a cada localidade para ambas as medidas. Para os homens, as notas de corte para força de preensão palmar variaram entre $0<\mathrm{IMC} \leq 23=17,67$ $\mathrm{Kg}_{\mathrm{f}} ; 23<\mathrm{IMC}<28=24,33 \mathrm{Kg}_{\mathrm{f}} ; 28 \leq \mathrm{IMC}<30=$ $22,00 \mathrm{Kg}_{\mathrm{f}}$ IMC $\leq 30=25,33 \mathrm{Kg}_{\mathrm{f}}$ (em Campina Grande) e $0<\mathrm{IMC} \leq 23=28,67 \mathrm{Kg}_{\mathrm{f}}$; $3<\mathrm{IMC}<$ $28=31,00 \mathrm{Kg}_{\mathrm{f}} ; 28 \leq \mathrm{IMC}<30=34,00 \mathrm{Kg}_{\mathrm{f}} ; \mathrm{IMC} \leq$ $30=28,67 \mathrm{Kg}_{\mathrm{f}}$ (em Poços de Caldas); para as 
mulheres a variação foi entre $0<\mathrm{IMC} \leq 23=$ $13,33 \mathrm{Kg}_{\mathrm{f}} ; 23<\mathrm{IMC}<28=14,67 \mathrm{Kg} ; 28 \leq \mathrm{IMC}$ $<30=13,33 \mathrm{Kg}$ f $\mathrm{IMC} \leq 30=14,67 \mathrm{Kg}_{\mathrm{f}}(\mathrm{em}$ Belém) e $0<\mathrm{IMC} \leq 23=17,33 \mathrm{Kg}$; $23<\mathrm{IMC}<$ $28=18,00 \mathrm{Kg}_{\mathrm{f}} ; 28 \leq \mathrm{IMC}<30=15,33 \mathrm{Kg}_{\mathrm{f}}$; IMC $\leq 30=18,50 \mathrm{Kg}_{\mathrm{f}}$ (em Ivoti). Para os homens, as notas de corte para velocidade da marcha variaram entre $0<$ alt $\leq 162 \mathrm{~cm}=5,60 \mathrm{~s}$ (em Ivoti) e $\geq$ $162 \mathrm{~cm}=6,07 \mathrm{~s}($ em Belém) e $0<$ alt $\leq 173 \mathrm{~cm}=$ $6,65 \mathrm{~s} \mathrm{e}>173 \mathrm{~cm}=4,52 \mathrm{~s}$ (Ivoti); para as mulheres a variação esteve entre $0<$ alt $\leq 147 \mathrm{~cm}=8,35 \mathrm{~s} \mathrm{e}$ $>147 \mathrm{~cm}=6,62 \mathrm{~s}$ (em Parnaíba) e $0<$ alt $\leq 160 \mathrm{~cm}$ $=6,29 \mathrm{~s}$ e $>160 \mathrm{~cm}=6,33 \mathrm{~s}$ (em Poços de Caldas).

Envolvimento social. Foi indicado pelo desempenho de 13 AAVD associadas à sociabilidade, ao lazer, à produtividade e à participação social e pelo desempenho independente de três AIVD que exigem autonomia cognitiva e manejo independente do ambiente social. Faziam parte do primeiro conjunto as seguintes atividades: fazer visitas, receber visitas, ir à igreja, participar de reuniões sociais, frequentar eventos culturais, guiar automóvel, fazer viagens de curtas distância e duração, fazer viagens de longas distância e duração; realizar trabalho voluntário, exercer trabalho remunerado, participar de diretorias ou conselhos, frequentar universidade da terceira idade e tomar parte em grupos de convivência ${ }^{11,12}$. Integravam o segundo conjunto as AIVD fazer compras, utilizar transporte e cuidar das finanças. As AAVD comportavam as respostas nunca fiz, parei de fazer ou ainda faço e as AIVD, informações sobre necessidade de ajuda (nenhuma, parcial ou total). Para cada idoso, o escore nas 16 atividades foi composto pela soma das AAVD mantidas, considerando-se as que cada idoso fazia anteriormente, e das AIVD desempenhadas com total independência. A distribuição dos escores foi expressa em porcentagens e dividida em três faixas: $<61 ; 61$ a $85 \%$ e $>85 \%$.

Satisfação referenciada a domínios. Mediante seis itens escalares, cada um com três intensidades (muito, mais ou menos e pouco) foi investigado o grau de satisfação em relação a seis domínios: memória, capacidade para resolver problemas cotidianos, amizades e relações familiares, ambiente (clima, barulho, poluição, atrativos e segurança), acesso a serviços de saúde e transportes $^{27}$.

\section{Análise de dados}

Os dados foram analisados por meio dos pacotes estatísticos SPSS $^{\circledast}$, versão 13.1, e SAS ${ }^{\circledast}$, versão 8.02. Os testes chi-quadrado e Exato de
Fisher foram usados para comparar frequências entre os grupos. Os testes de Mann Whitney e Kruskal-Wallis / post hoc de Dunn, foram usados para comparar as médias das medidas de doenças, força muscular e envolvimento social. Foi derivado um indicador de consistência interna entre as pontuações nos domínios de satisfação (alfa de Cronbach). O teste de Spearman foi adotado para as análises das correlações entre envolvimento social e capacidade funcional. A margem de erro aceita para todas as medidas foi indicada por $p \leq 0,05$.

\section{Resultados}

A idade média dos participantes foi 72,2 \pm 5,5 anos (variação de 65 a 96 anos); 65,7\% eram mulheres. A renda familiar média foi 3,9 $\pm 4,9$ SM. Apenas 11,3\% dos idosos não tinham doenças crônicas, $49,7 \%$ tinham 1 ou 2 e 39,0\% tinham 3 ou mais; $17 \%$ pontuaram para baixa força de preensão e 16,9\% para lentidão da marcha; $50 \%$ tiveram escores entre $61 \%$ e $85 \%, 24,2 \%$ pontuaram abaixo de $61 \%$ e $25,5 \%$ acima de $85 \%$ em envolvimento social. As AAVD desempenhadas por mais idosos foram: receber e fazer visitas (respectivamente $90,8 \%$ e $76,5 \%$ ) e ir à igreja $(88,4 \%)$. As atividades interrompidas por mais idosos foram trabalho $(60,7 \%)$, viagens longas $(30,6 \%)$, festas e reuniões $(27,5 \%)$ e participar de eventos culturais $(27,5 \%)$. As menos desempenhadas anteriormente envolviam participar de universidade da terceira idade $(86,2 \%)$, diretorias e conselhos $(70 \%)$, centros de convivência $(61,1 \%)$ e trabalho voluntário $(59,7 \%)$ e guiar automóvel $(66,9 \%)$. Os idosos declararam-se muito satisfeitos com as relações familiares e de amizade $(78,4 \%)$, a capacidade de resolver problemas cotidianos $(71,3 \%)$, os serviços de transporte $(55,5 \%)$, os serviços de saúde $(52,8 \%)$, as condições do ambiente (52\%) e a memória $(50 \%)$. Vinte e um por cento relataram estar pouco satisfeitos com o ambiente, $18 \%$ com os serviços de saúde, $14 \%$ com os transportes e 14\% com a memória. A consistência interna entre as medidas de satisfação referenciada a domínios foi moderada $(\alpha=0,663)$, indicando que eles são relativamente independentes entre si.

O número médio de doenças crônicas foi maior entre as mulheres, em comparação com os homens. O tempo de marcha das mulheres foi significativamente maior do que o dos homens e a força de preensão delas foi significativamente mais baixa do que a deles. No entanto, o escore 
das mulheres em envolvimento social foi mais alto do que o dos homens. Não foram observadas diferenças estatisticamente significantes entre as médias dos homens e das mulheres nas medidas de satisfação referenciada a domínios, com exceção de satisfação com os transportes, em que a médias das mulheres foi menor do que a dos homens (Tabela 1).

Não foram observadas diferenças entre os grupos etários com respeito ao número de doenças. Os idosos de 80 anos e mais tiveram médias significantemente mais altas em tempo de marcha e mais baixas em força de preensão e envolvimento social do que os grupos mais jovens. Os idosos de 65 a 69 anos pontuaram significativamente mais alto do que os demais em satisfação para resolver problemas cotidianos, enquanto que os mais velhos tiveram médias significativamente mais altas em satisfação com o ambiente e com os serviços de saúde (Tabela 2).

Os idosos com renda inferior a $1 \mathrm{SM}$ foram o grupo com mais doenças crônicas e os com renda superior a 10 SM, o grupo com menos doenças entre todos os grupos de renda. Os grupos com renda superior a 10 SM destacaram-se entre os demais por apresentarem melhor pontuação em capacidade funcional, envolvimento social e satisfação com os transportes e com os serviços de saúde. Os grupos com renda de $\leq 1$ a 3 SM tiveram as menores médias em satisfação com a memória e com a capacidade de resolver problemas cotidianos. Os de renda superior a 10 SM

Tabela 1. Comparação entre as médias observadas em número de doenças, capacidade funcional e satisfação referenciada a domínios, considerando-se a variável gênero. Estudo Fibra, polo Unicamp. Idosos, 2008-

2009.

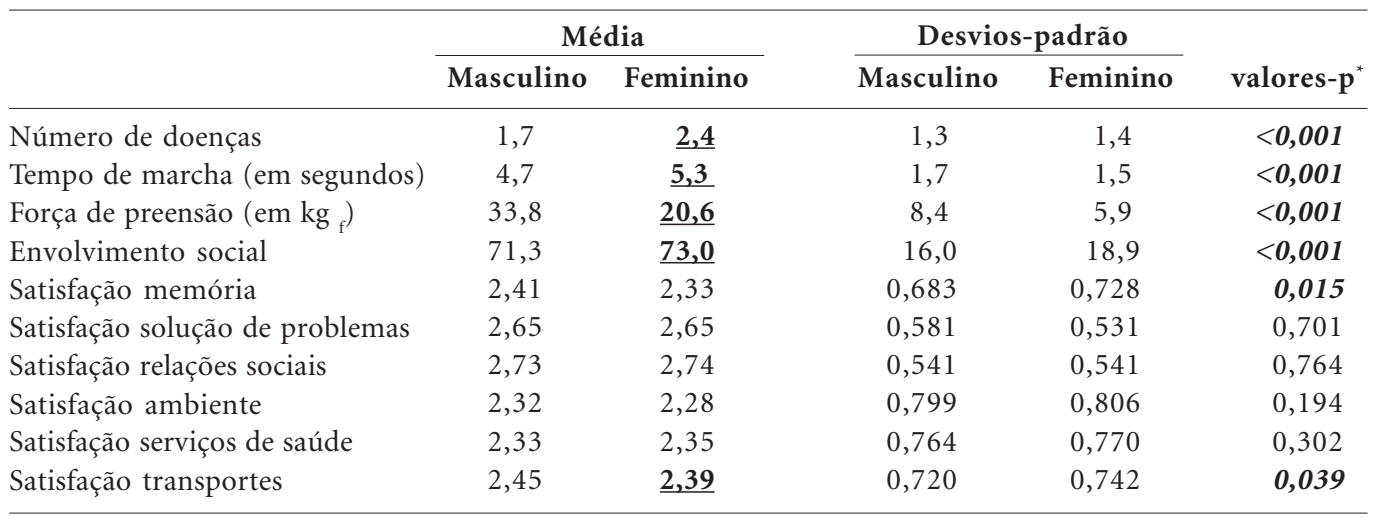

Homens $=847$; mulheres $=1625 .{ }^{*}$ Teste U de Mann-Whitney

Tabela 2. Comparação entre as médias observadasem número de doenças, capacidade funcional e satisfação referenciada a domínios, considerando-se a variável idade. Estudo Fibra, polo Unicamp. Idosos, 2008-2009.

\begin{tabular}{|c|c|c|c|c|c|c|c|c|c|}
\hline & \multicolumn{4}{|c|}{ Média } & \multicolumn{4}{|c|}{ Desvios-padrão } & \multirow[b]{2}{*}{ valores-p } \\
\hline & $65-69$ & $70-74$ & $75-79$ & $80 \mathrm{e}+$ & $65-69$ & $70-74$ & $75-79$ & $80 \mathrm{e}+$ & \\
\hline Número de doenças & 2,1 & 2,2 & 2,4 & 2,1 & 1,5 & 1,4 & 1,5 & 1,4 & 0,084 \\
\hline Tempo de marcha (em segundos) & 4,8 & 5,0 & 5,3 & $\underline{5,8}$ & 1,3 & 1,8 & 1,5 & 1,9 & $<0,001$ \\
\hline Força de preensão $\left(\mathrm{em} \mathrm{kg}_{\mathrm{f}}\right)$ & 26,2 & 26,1 & 23,0 & $\underline{22,1}$ & 9,8 & 9,1 & 8,2 & 8,4 & $<0,001$ \\
\hline Envolvimento social & 75,6 & 72,4 & 70,9 & 64,6 & 16,8 & 17,6 & 18,0 & 21,2 & $<0,001$ \\
\hline Satisfação memória & 2,4 & 2,3 & 2,4 & $\overline{2,3}$ & 0,7 & 0,7 & 0,7 & 0,7 & 0,274 \\
\hline Satisfação solução de problemas & $\underline{2,7}$ & 2,6 & 2,6 & 2,6 & 0,5 & 0,6 & 0,6 & 0,6 & 0,042 \\
\hline Satisfação relações sociais & $\overline{2,7}$ & 2,7 & 2,7 & 2,8 & 0,5 & 0,5 & 0,5 & 0,5 & 0,339 \\
\hline Satisfação ambiente & 2,2 & 2,2 & 2,3 & $\underline{2,5}$ & 0,8 & 0,8 & 0,8 & 0,7 & 0,003 \\
\hline Satisfação serviços de saúde & 2,3 & 2,3 & $\underline{2,5}$ & $\underline{2,4}$ & 0,7 & 0,8 & 0,7 & 0,7 & 0,005 \\
\hline Satisfação transportes & 2,4 & 2,4 & 2,4 & 2,4 & 0,7 & 0,7 & 0,7 & 0,8 & 0,388 \\
\hline
\end{tabular}

$65-69=941 ; 70-74=773 ; 75-79=467 ; 80$ anos $\mathrm{e}+=291 .{ }^{*}$ valor de $\mathrm{p}$ referente ao Teste Kruskal-Wallis 
revelaram-se os mais satisfeitos com os serviços de saúde e os transportes (Tabela 3).

Mais mulheres do que homens manifestaram insatisfação com a memória e mais idosos de 65 a 74 anos do que dos outros grupos etários pontuaram para insatisfação com o ambiente e com os serviços de saúde. Baixa força de preensão e lentidão da marcha relacionaram-se com baixa satisfação com a capacidade de resolver problemas cotidianos e com as relações sociais. Por outro lado, renda familiar superior a $3 \mathrm{SM}$, não ter nenhuma ou ter 1 ou 2 doenças e ter alto escore de envolvimento social relacionaram-se com alta satisfação com a memória. Não ter nenhuma doença, não pontuar para baixa força de preensão e nem para lentidão da marcha e ter alto escore em AAVD associou-se com alta satisfação com a capacidade de resolver problemas. Essas relações se repetiram para satisfação com as relações sociais. Não ter doenças e ter alto escore em AAVD relacionou-se com alta satisfação em relação ao ambiente. Ter renda superior a $10 \mathrm{SM}$, não ter doenças, não ter lentidão da marcha e ter alto escore em AAVD associou-se com alta satisfação com os transportes. Finalmente, mais idosos com renda superior a $10 \mathrm{SM}$ do que dos outros grupos de renda, e mais idosos com alto escore doque com escores baixos ou moderados em AAVD avaliaram-se como satisfeitos em relação aos serviços de saúde. Em suma, as condições de saúde, capacidade funcional e envolvimento social mostraram-se mais relacionadas com satisfação referenciada a domínios do que as variáveis gênero, idade e renda familiar (Tabela 4).

Foram calculadas as correlações entre os escores em AAVD e as pontuações nos testes de capacidade funcional para a amostra total e para os grupos de gênero e de idade. Os valores do teste de preensão palmar guardaram relação positiva e estatisticamente significativa com os escores em AAVD na amostra como um todo, nos grupos de homens e de mulheres e no grupo de 65 a 69 anos, indicando que quanto mais alta a pontuação em força de preensão manual, maior o envolvimento em AAVD. Em contrapartida, quanto maior a lentidão da marcha, menor o envolvimento em AAVD em todos os grupos considerados, como indicam os valores igualmente baixos, mas estatisticamente significativos obtidos nos testes de correlação (Tabela 5).

\section{Discussão}

No Estudo Bambuí ${ }^{28}$, $69 \%$ dos idosos relataram ter pelo menos uma doença crônica, sendo esta proporção maior entre as mulheres $(74,5 \%)$ do que entre os homens $(62,2 \%)$. No Cardiovascular Health Study (CHS $)^{24}$ verificou-se que apenas $18,5 \%$ dos idosos não referiram doença crônica. Em consonância com esses dados, cerca de 90\% dos idosos deste estudo relatou ter pelo menos uma doença crônica. A frequência de idosos que pontuaram para baixa força de preensão foi semelhante às prevalências dessa condição em estudos internacionais com idosos independentes recrutados na comunidade. No CHS $^{24}$ foi de $20 \%$ e no Women's Health and Aging Study (WHAS) foi de 20,8\% ${ }^{29}$; no estudo brasileiro de Breda ${ }^{30}$, com idosos atendidos pelo Programa de Saúde da Família, foi de $20 \%$. Resultados semelhantes foram observados para as medidas de velocidade de

Tabela 3. Comparação entre as médias observadas em número de doenças, capacidade funcional e satisfação referenciada a domínios, considerando-se o nível de renda familiar. Estudo Fibra, polo Unicamp. Idosos, 2008-2009.

\begin{tabular}{|c|c|c|c|c|c|c|c|c|c|c|c|}
\hline & \multicolumn{5}{|c|}{ Médias } & \multicolumn{5}{|c|}{ Desvios-padrão } & \multirow{2}{*}{$\begin{array}{c}\text { valores- } \\
\mathbf{p}^{*}\end{array}$} \\
\hline & $<1$ & $1,1-3,0$ & $3,1-5,0$ & $5,1-10,0$ & $>10$ & $<1$ & $1,1-3,0$ & $3,1-5,0$ & $5,1-10,0$ & $>10$ & \\
\hline Número de doenças & $\underline{2,4}$ & 2,2 & 2,1 & 2,2 & $\underline{1,9}$ & 1,5 & 1,5 & 1,4 & 1,4 & 1,9 & 0,008 \\
\hline Tempo de marcha (em segundos) & $\overline{5,3}$ & 5,3 & 5,0 & 5,0 & $\overline{4,5}$ & 1,5 & 1,5 & 1,4 & 2,5 & 4,5 & $<0,001$ \\
\hline Força de preensão $\left(\mathrm{em} \mathrm{kg}_{\mathrm{f}}\right)$ & 23,0 & 24,9 & 26,6 & 26,6 & $\underline{29,2}$ & 8,0 & 8,9 & 10,0 & 9,7 & 29,2 & $<0,001$ \\
\hline Envolvimento social & 71,0 & 70,5 & 74.5 & 72,3 & $\underline{77,8}$ & 19,4 & 18,8 & 17,2 & 17,1 & 77,9 & $<0,001$ \\
\hline Satisfação memória & $\underline{2,2}$ & $\underline{2,3}$ & 2,4 & 2,4 & 2,4 & 0,7 & 1,0 & 1,0 & 3,0 & 0,6 & 0,001 \\
\hline Satisfação solução de problemas & $\underline{2,6}$ & $\underline{2,6}$ & 2,7 & 2,7 & 2,7 & 0,6 & 1,0 & 1,0 & 3,0 & 1,5 & 0,032 \\
\hline Satisfação relações sociais & 2,7 & 2,7 & 2,8 & 2,7 & 2,7 & 0,5 & 1,0 & 1,0 & 3,0 & 0,5 & 0,712 \\
\hline Satisfação ambiente & 2,2 & 2,3 & 2,3 & 2,3 & 2,3 & 0,8 & 1,0 & 1,0 & 3,0 & 0,8 & 0,357 \\
\hline Satisfação serviços de saúde & 2,2 & 2,3 & 2,4 & 2,4 & $\underline{2,5}$ & 0,8 & 1,0 & 1,0 & 3,0 & 0,8 & 0,002 \\
\hline Satisfação transportes & 2,2 & 2,3 & 2,5 & 2,5 & $\overline{2,6}$ & 0,7 & 1,0 & 1,0 & 3,0 & 0,6 & $<0,001$ \\
\hline
\end{tabular}

$65-69=941 ; 70-74=773 ; 75-79=467 ; 80$ anos $\mathrm{e}+=291 .{ }^{*}$ valor de p referente ao Teste Kruskal-Wallis 
Tabela 4. Idosos por gênero, idade, renda familiar, doenças crônicas autorrelatadas, força de preensão palmar, velocidade de marcha e envolvimento social conforme os graus de satisfação referenciada a domínios. Estudo FIBRA Unicamp, Campinas, Brasil. Idosos, 2008-2009.

\begin{tabular}{|c|c|c|c|c|c|c|c|c|}
\hline \multirow{3}{*}{ Gênero } & \multicolumn{3}{|c|}{ Memória } & \multirow[b]{2}{*}{ valor $p$} & \multicolumn{3}{|c|}{ Resolver problemas } & \multirow[b]{2}{*}{ valor $p$} \\
\hline & Pouco & MM & Muito & & Pouco & MM & Muito & \\
\hline & & & & 0,013 & & & & $=0,420$ \\
\hline Masculino & 11,2 & 36,1 & 52,6 & & 5,4 & 24,3 & 70,2 & \\
\hline Feminino & 15,3 & 36,1 & 48,5 & & 6,0 & 22,1 & 71,7 & \\
\hline Idade & & & & 0,439 & & & & $=0,210$ \\
\hline 65-69 & 13,5 & 34,7 & 51,7 & & 4,7 & 22,3 & 72,9 & \\
\hline $70-74$ & 13,4 & 38,0 & 48,5 & & 5,6 & 21,8 & 72,4 & \\
\hline $75-79$ & 13,2 & 36,1 & 50,5 & & 7,7 & 24,8 & 67,4 & \\
\hline $80+$ & 17,5 & 35,4 & 47 & & 6,8 & 24,4 & 68,7 & \\
\hline Renda Familiar & & & & 0,006 & & & & $=0,164$ \\
\hline$\leq 1 \mathrm{SM}$ & $\underline{15,6}$ & 42,8 & 41,5 & & 7,6 & 26,2 & 66,1 & \\
\hline $1-3 \mathrm{SM}$ & $\underline{15,6}$ & 36,9 & 47,4 & & 6,5 & 25,1 & 68,2 & \\
\hline $3,1-5 \mathrm{SM}$ & 11,8 & 34,6 & $\underline{53,5}$ & & 5,7 & 20,3 & 73,8 & \\
\hline $5,1-10 \mathrm{SM}$ & 10,2 & 37,7 & $\underline{52}$ & & 4,4 & 20,8 & 74,7 & \\
\hline$>10 \mathrm{SM}$ & 7,5 & 34,0 & $\underline{58,3}$ & & 3,7 & 22,7 & 73,4 & \\
\hline Doenças & & & & $<0,001$ & & & & $<0,001$ \\
\hline 0 & 12,1 & 31,1 & $\underline{56,6}$ & & 3,5 & 16,8 & $\underline{79,5}$ & \\
\hline $1-2$ & 11,6 & 35,2 & $\underline{53,1}$ & & 5,0 & 21,5 & 73,4 & \\
\hline $3+$ & $\underline{17,3}$ & 38,7 & 43,9 & & $\underline{7,5}$ & 26,3 & 66,1 & \\
\hline BFpreensão & & & & 0,011 & & & & $<0,001$ \\
\hline Não & 13,0 & 37,1 & 49,8 & & 4,9 & 22,1 & 72,9 & \\
\hline Sim & $\underline{17,9}$ & 31,6 & 50,3 & & $\underline{10,3}$ & 26,3 & 63,3 & \\
\hline Lmarcha & & & & 0,152 & & & & $<0,001$ \\
\hline Não & 13,4 & 35,8 & 50,7 & & 4,7 & 21,2 & 74,0 & \\
\hline Sim & $\underline{16,3}$ & 37,3 & 46,2 & & $\underline{11,3}$ & 31,0 & 57,5 & \\
\hline Env social & & & & $<0,001$ & & & & $<0,001$ \\
\hline$<61 \%$ & 18,5 & 38,9 & 42,4 & & $\underline{10,2}$ & 31,6 & 58,1 & \\
\hline $61-85 \%$ & 14,3 & 36,5 & 49,1 & & 4,8 & 22,7 & $\underline{72,4}$ & \\
\hline$>85 \%$ & 8,7 & 32,4 & $\underline{58,8}$ & & 3,8 & 14,9 & $\underline{81,3}$ & \\
\hline
\end{tabular}

marcha, cuja prevalência de baixo desempenho nos estudos do grupo de Fried foi de $20 \%{ }^{24,29}$.

As condições de saúde e funcionalidade são fortemente relacionadas à renda familiar, à escolaridade, à idade e a sexo $0^{10,24,29,30}$. No Brasil, os efeitos desses fatores associam-se a dificuldades de acesso a serviços de saúde e à escassez de informações e de oportunidades para a manutenção das condições físicas e funcionais na velhi$\mathrm{ce}^{31,32}$. Os papéis sociais desempenhados ao longo da vida pelas mulheres acarretam-lhes desvantagens sociais que se acumulam e se manifestam como doença e incapacidade ${ }^{9,10,27,32-34}$. Os dados deste estudo sobre relações entre ser mulher, ter maior número de doenças crônicas e pontuar mais baixo em testes de capacidade funcional refletem essas relações.

No entanto, as mulheres do Estudo Fibra tiveram média significativamente mais alta do que os homens em envolvimento social, provavelmente em virtude do imperativo de cumprir normas sociais associadas ao cuidado e ao apoio social no âmbito da família e das relações sociais $^{34}$. Para desempenhar os papéis e as tarefas associados a esse imperativo, mulheres com comorbidades e incapacidades tendem a realizar adaptações e compensações, por exemplo, atuando mais lentamente, parcelando as tarefas, submetendo-se a menos exigências de perfeição, recrutando ajuda e diminuindo o repertório de atividades feitas ao mesmo tempo ${ }^{35,36}$. Em virtude da maior exposição às experiências relevantes, as mulheres desenvolvem mais habilidades sociais, maior facilidade para expressar e compreender sentimentos e menos restrições a pedir ajuda. Enquanto isso, os homens envolvem-se quase que exclusivamente em atividades laborais e têm menos chance de desenvolver habilidades sociais 
Tabela 4. continuação

\begin{tabular}{|c|c|c|c|c|c|c|c|c|}
\hline \multirow{3}{*}{ Gênero } & \multicolumn{3}{|c|}{ Relações sociais } & \multirow[b]{2}{*}{ valor $p$} & \multicolumn{3}{|c|}{ Ambiente } & \multirow[b]{2}{*}{ valor $p$} \\
\hline & Pouco & MM & Muito & & Pouco & MM & Muito & \\
\hline & & & & 0,916 & & & & $=0473$ \\
\hline Masculino & 4,8 & 17,2 & 77,9 & & 20,6 & 25,8 & 53,4 & \\
\hline Feminino & 4,5 & 16,8 & 78,5 & & 21,8 & 27,2 & 50,8 & \\
\hline Idade & & & & 0,307 & & & & $=0,005$ \\
\hline 65-69 & 5,4 & 16,2 & 78,3 & & $\underline{22,4}$ & 27,9 & 49,6 & \\
\hline $70-74$ & 3,6 & 17,7 & 78,9 & & $\underline{24,5}$ & 25,4 & 49,9 & \\
\hline $75-79$ & 5,3 & 18,6 & 76,0 & & 18,4 & 27,1 & 54,3 & \\
\hline $80+$ & 4,4 & 14,7 & 80,7 & & 14,7 & 25,7 & $\underline{59,4}$ & \\
\hline Renda Familiar & & & & 0,786 & & & & $=0,362$ \\
\hline$\leq 1 \mathrm{SM}$ & 4,2 & 19,4 & 76,2 & & 23,3 & 32,2 & 44,4 & \\
\hline $1-3 \mathrm{SM}$ & 4,5 & 18,2 & 77,2 & & 20,8 & 26,4 & 52,7 & \\
\hline $3,1-5 \mathrm{SM}$ & 5,3 & 15,0 & 79,6 & & 22,2 & 24,6 & 53 & \\
\hline $5,1-10 \mathrm{SM}$ & 4,7 & 14,6 & 80,5 & & 18,6 & 29,6 & 51,6 & \\
\hline$>10 \mathrm{SM}$ & 4,5 & 16,6 & 78,7 & & 22,7 & 25,7 & 51,5 & \\
\hline Doenças & & & & 0,381 & & & & $=0,015$ \\
\hline 0 & 3,9 & 17,2 & 78,8 & & 17,9 & 22,5 & $\underline{59,5}$ & \\
\hline $1-2$ & 4,4 & 15,7 & 79,8 & & 20,1 & $\underline{27,8}$ & $\overline{52,0}$ & \\
\hline $3+$ & 5,0 & 18,5 & 76,3 & & $\underline{24,0}$ & $\underline{26,7}$ & 49,2 & \\
\hline BFpreensão & & & & 0,009 & & & & $=0,561$ \\
\hline Não & 4,4 & 15,9 & $\underline{79,5}$ & & 20,9 & 26,9 & 52,1 & \\
\hline Sim & 5,2 & 21,8 & 72,9 & & 23,2 & 25,6 & 51 & \\
\hline Lmarcha & & & & 0,038 & & & & $=0,798$ \\
\hline Não & 4,3 & 16,3 & $\underline{79,3}$ & & 21,0 & 26,6 & 52,3 & \\
\hline Sim & 6,0 & 20,2 & $\overline{73,7}$ & & 22,1 & 27,2 & 50,6 & \\
\hline Env social & & & & $<0,001$ & & & & $=0,024$ \\
\hline$<61 \%$ & 4,8 & 23,5 & 71,5 & & 25,4 & 26,2 & 48,3 & \\
\hline $61-85 \%$ & 4,7 & 15,4 & $\underline{79,7}$ & & 21,2 & 26,8 & 51,9 & \\
\hline$>85 \%$ & 3,9 & 13,7 & $\underline{82,2}$ & & 17,7 & 27,2 & $\underline{54,9}$ & \\
\hline
\end{tabular}

continua

ou competências de regulação emocional, o que os coloca em desvantagem na velhice, quando perdem o papel ocupacional em virtude da aposentadoria $^{34}$.

Os idosos de 80 anos e mais apresentaram pior desempenho nas medidas de capacidade funcional do que os mais jovens. O declínio da força muscular ocorre devido às alterações quantitativas e qualitativas das fibras musculares, que se fazem acompanhar pela substituição do tecido muscular por tecido adiposo e pela redução da capacidade de recrutamento neural. Tais alterações podem repercutir no desempenho de atividades sociais, produtivas, de lazer e organizacionais complexas e na execução de tarefas da vida prática. As mudanças fisiológicas do envelhecimento ocasionam aumento na probabilidade de o idoso apresentar lentidão da marcha, problemas de mobilidade e equilíbrio e baixa força de preensão palmar. A sarcopenia, as comorbidades, as alterações em funcionalidade, o uso de medicamentos, a depressão e o baixo senso de autoeficácia derivado de experiências de insucesso associam-se à diminuição do envolvimento social e da satisfação $0^{4,5,9,13,16,17,37-40}$.

Os idosos mais velhos apresentaram os escores mais baixos na medida de envolvimento social. Parte dos idosos pode ter diminuído seu envolvimento em atividades sociais realizadas fora de casa, porque o ambiente físico impõe barreiras ao seu desempenho ${ }^{35}$, aumenta sua insegurança ou sua prudência e afeta seu senso de autoeficácia $^{15}$. Parte deles terá diminuído o envolvimento social em virtude do processo de seletividade socioemocional: os idosos tendem a abandonar atividades que demandam mais esforços de adaptação ou que são menos significativas, em favor de outras que lhe trazem conforto emocional ${ }^{14}$.

As mulheres superaram os homens em frequência de baixa satisfação com a memória, confirmando dados da literatura que apontam que elas têm senso de autoeficácia mais baixo em rela- 
Tabela 4. continuação

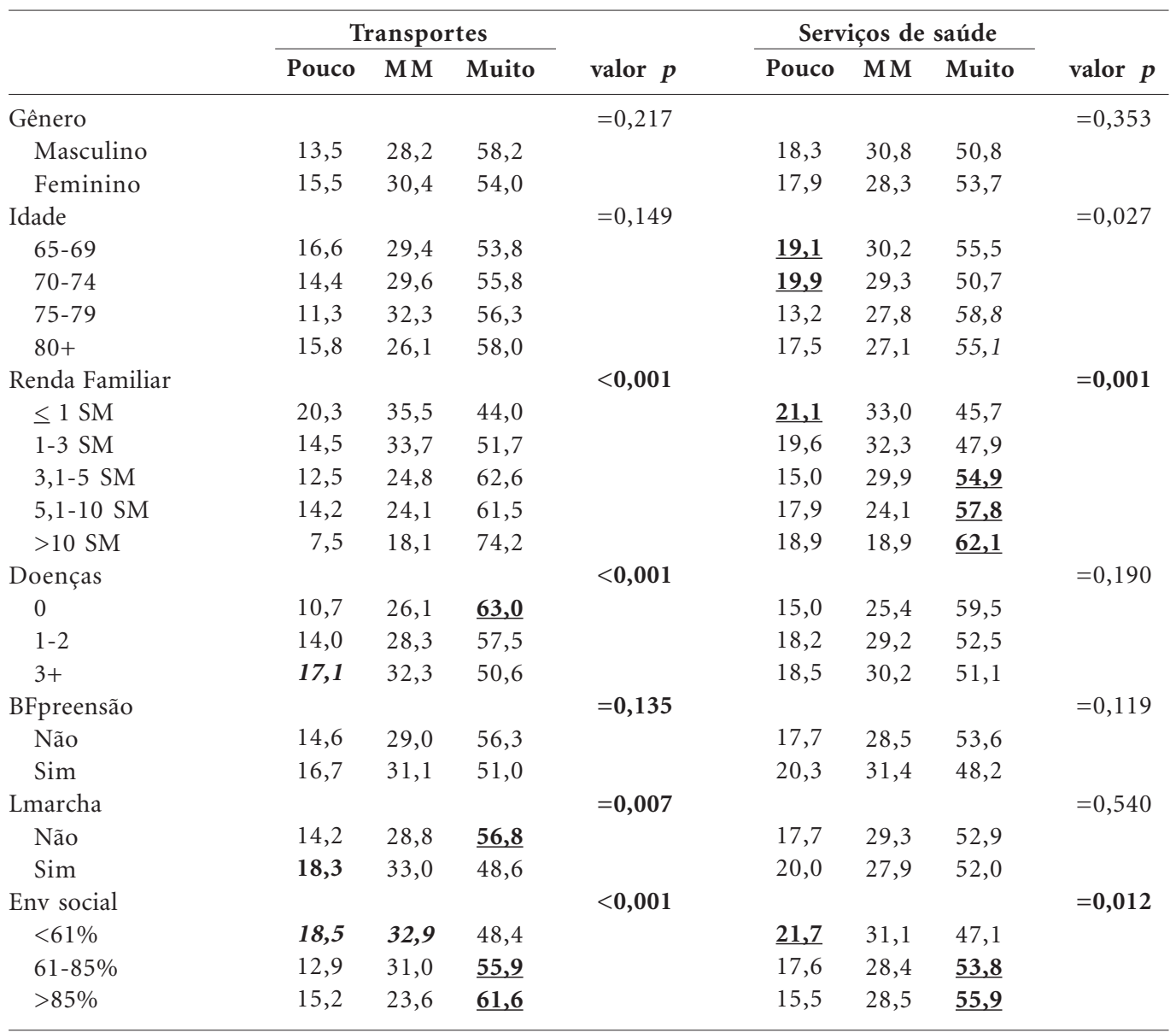

Tabela 5. Valores das correlações entre as variáveis indicativas de envolvimento social e de capacidade funcional na amostra total, conforme gênero e idade. Fibra, polo Unicamp, Brasil. Idosos, 20082009.

\begin{tabular}{lcc}
\hline & $\begin{array}{c}\text { Força } \\
\text { de preensão }\end{array}$ & $\begin{array}{c}\text { Velocidade } \\
\text { da marcha }\end{array}$ \\
\hline Amostra total & rho $=0,047$ & rho $=-0,216$ \\
& $\boldsymbol{p}=\mathbf{0 , 0 1 8}$ & $\boldsymbol{p}<\mathbf{0 , 0 0 1}$ \\
Homens & rho $=0,130$ & rho $=-0178$ \\
& $\boldsymbol{p}=\mathbf{0 , 0 0 1}$ & $\boldsymbol{p}<\mathbf{0 , 0 0 1}$ \\
Mulheres & rho $=0,138$ & rho $=-0,265$ \\
& $\boldsymbol{p}<\mathbf{0 , 0 0 1}$ & $\boldsymbol{p}<\mathbf{0 , 0 0 1}$ \\
65-69 anos & rho $=0,0280$ & rho $=-, 0191$ \\
& $p=0,392$ & $\mathrm{p}<0,001$ \\
70-74 anos & rho $=0,011$ & Rho $=-0,165$ \\
& $p=0,741$ & $\boldsymbol{p}<\mathbf{0 , 0 0 1}$ \\
75-79 anos & rho $=0,069$ & rho $=-0,192$ \\
& $p=0,136$ & $\boldsymbol{p}<\mathbf{0 , 0 0 1}$ \\
80 anos e + & rho $=-0,022$ & Rho $=-0,272$ \\
& $p=0,701$ & $\boldsymbol{p}<\mathbf{0 , 0 0 1}$ \\
\hline
\end{tabular}

ção à memória e à cognição do que os homens e que seu desempenho cognitivo de fato é pior do que o deles. Tais ocorrências têm sido explicadas por prejuízos educacionais e econômicos acumulados ao longo da vida e por comorbidades, depressão, autoavaliação negativa de saúde e incapacidade funcional, que afetam mais as mulheres idosas do que os homens idosos ${ }^{34,37-39}$.

Entre os idosos mais jovens (65-69 anos) ocorreram percentuais mais altos de respostas de insatisfação com o ambiente e com os serviços de saúde, possivelmente porque têm mais contato com esses domínios, ao contrário dos mais velhos, que são menos desafiados porque já restringiram seus contatos e têm menos oportunidades de arriscar-se nesses contextos. Na fase inicial da velhice, as pessoas tendem a experimentar a necessidade de compatibilizar as perdas em capacidades físicas com as exigências de atividade e de desempenho de papéis adultos. Nesse momento, o ambiente pode ser encarado como barreira 
ou facilitador, o que se reflete nos níveis de satisfação ${ }^{35,38,39}$. Possivelmente, a satisfação com os serviços de saúde entre os idosos mais velhos não reflete sua boa qualidade, mas sim baixa escolaridade e baixa renda, que os torna menos críticos e menos exigentes com relação à existência de barreiras arquitetônicas e com a qualidade técnica e humana do atendimento. Foram observadas frequências mais elevadas de idosos muito satisfeitos com os serviços de saúde entre os de maior renda do que entre os com menor renda, provavelmente porque aqueles desfrutam de melhor atendimento nos dois setores ${ }^{33}$. Há mais idosos com renda mais alta satisfeitos com a memória do que os de baixa renda, possivelmente porque aqueles são mais protegidos pela escolaridade e pela estimulação oriunda do trabalho e do ambiente do que estes últimos ${ }^{31,33}$.

Houve um percentual significativamente maior de idosos sem ou com 1 ou 2 doenças crônicas entre os que pontuaram para alta satisfação com a memória, a capacidade de resolver problemas do dia-a-dia, os serviços de saúde e os transportes. Um percentual maior de idosos com baixa força de preensão em comparação com os que não pontuaram para esse tipo de perda descreveu-se como pouco satisfeitos com a memória, a capacidade de resolver problemas do dia-a-dia e as amizades e relações sociais. Um percentual alto e significativamente maior de idosos sem perda de força de preensão e sem lentidão da marcha declarou-se muito satisfeito com relação a esses domínios da satisfação. Os dados sugerem que os idosos mais fortes e mais ágeis tendem a estar mais satisfeitos com diversos domínios da vida do que os mais fracos e os mais lentos, provavelmente porque retém maior funcionalidade ${ }^{37-40}$. $O$ fato de o envolvimento em AAVD ter se mostrado relacionado à pontuação mais elevada em todos os domínios de satisfação reforça a noção de que as condições de saúde e de função física tendem a afetar o bem-estar subjetivo. Maior envolvimento social é fator protetor em relação a perdas funcionais e cognitivas e propicia a existência de redes de suporte psicossocial e material que contribuem para a maior satisfação nos domínios da memória, da capacidade para resolver problemas e das relações sociais ${ }^{37-40}$.

As distribuições de frequência relativas às AAVD que os idosos haviam deixado de desempenhar, ainda desempenhavam e nunca haviam desempenhado sugerem que o envolvimento social dessas pessoas sofre não apenas a influência das condições de saúde e capacidade funcional, como também da atribuição de papéis de gênero e idade e de variáveis socioeconômicas. De fato, frequentar programas de extensão oferecidos pelas universidades e ir a centros de convivência dependem da disponibilidade desses recursos. $\mathrm{Na}$ eventualidade de os recursos estarem presentes, não é qualquer idoso que pode participar dessas atividades, considerando-se que as instituições estabelecem esquemas de horário e ofertas não necessariamente compatíveis com os repertórios de quaisquer homens ou mulheres idosos, com diferentes ocupações anteriores, níveis de escolaridade e renda, compromissos familiares, hábitos e interesses.

O voluntariado ainda não é uma atividade tão disseminada no Brasil como em outros países e também faz exigências aos seus participantes. A participação em diretorias e conselhos integra uma classe de comportamentos relacionada ao exercício da cidadania, que depende de exposição anterior, motivação e características de personalidade, e ainda é uma atividade com forte vinculação ao gênero masculino. Guiar automóvel depende do poder aquisitivo das pessoas e, para as pessoas que hoje tem mais de 65 anos, também tem forte vinculação ao gênero masculino. A interrupção do trabalho remunerado tem forte relação com a aposentadoria por idade, combinada ou não com tempo de serviço, e com aposentadoria por invalidez. Receber visitas, ir à igreja e fazer visitas são atividades realizadas no domicílio ou nas vizinhanças, ao passo que viajar e ir a festas e reuniões pode envolver deslocamentos maiores e, assim, mais exigência física, cognitiva, motivacional. Além disso, a continuidade das atividades desempenhadas fora do ambiente doméstico e da vizinhança pode relacionar-se com motivação e com escolhas pessoais.

A abordagem da saúde do idoso requer uma perspectiva multidimensional e integral dos fenômenos saúde, funcionalidade, envolvimento social e bem-estar subjetivo. O envolvimento social evidenciado pela participação em atividades sociais, produtivas, de lazer e organizacionais é um bom indicador de capacidade funcional, de motivação para a atividade e para o contato social, e de cognição preservada, motivo pelo qual deve merecer atenção na avaliação do idoso. A avaliação precoce e precisa de limitações nessas atividades, que precedem as perdas em AIVD e em $A B V D$, é de grande valia à promoção e à manutenção da qualidade de vida até idade avançada. O envolvimento social e a satisfação são indicadores de envelhecimento saudável e elementos protetores com relação a perdas funcionais, isolamento e depressão na velhice. 


\section{Conclusões}

Neste trabalho foram observadas relações positivas entre saúde, capacidade funcional, envolvimento social e satisfação, assim como foram observadas relações entre idade avançada, capacidade funcional prejudicada, menor envolvimento social e menor satisfação com relação a domínios específicos do funcionamento. Investigações sobre o uso do tempo e sobre as preferências e os motivos dos idosos para envolvimento em atividades sociais, considerando-se gênero, idade, nível socioeconômico e cognição, poderão contribuir para a melhor compreensão das relações in-

\section{Colaboradores}

AL Neri planejou e coordenou a pesquisa do Estudo Fibra - polo Unicamp, planejou este estudo e acompanhou JM Pinto na análise e na interpretação de dados e na redação do artigo.

\section{Agradecimentos}

Monica Sanches Yassuda auxiliou na construção do projeto de pesquisa do Estudo Fibra, polo Unicamp e, junto com Jose Guilherme de Arruda Moura, Ludgleydson F. de Araújo, Maria do Carmo Eulálio, Benedita Edina Cabral, Maria Eliane Catunda de Siqueira e coordenou as equipes de recrutamento e coleta de dados nas cidades parceiras. Efigênia Passarelli Mantovani, Andrea Cristina Garofe Fortes-Burgos treinaram as equipes de recrutamento e análise de dados das sete localidades e auxiliaram na construção dos manuais de treinamento. Flavia Silva Arbex Borim, Stella Vital de Souza Torres e Débora Dias da Silva Harmitt coordenaram a coleta de dados em Campinas, junto com Andréa Fortes e Efigênia Mantovani. Natália de Oliveira Rodrigues coordenou a equipe de digitação de dados integrada por Juliana Martins, Veridiana Menezes, Anderson Vieira dos Santos, Kally Chung, Cristiane de Rezende Jayme, Cecília de Moraes Barbosa, Mariane Barroso Pereira e Cristina Mandau Okuni Cá. Ao Conselho Nacional de Desenvolvimento Científico e Tecnológico (CNPq) e Coordenação de Aperfeiçoamento de Pessoal de Nível Superior (CAPES), pelo apoio financeiro. vestigadas. Estudos longitudinais serão úteis para esclarecer como as relações mudam ao longo do envelhecimento. $\mathrm{O}$ acompanhamento de idosos menos independentes e saudáveis em termos físicos e cognitivos, em comparação com grupos com melhor qualidade de vida, será útil para esclarecer as questões levantadas por este trabalho. $\mathrm{O}$ tema é de alta relevância, pois a satisfação dos idosos nos diferentes domínios da vida e seu envolvimento com vários tipos de atividades podem ser tomados como indicadores de qualidade de vida na velhice, de necessidades da população idosa e da eficácia das políticas sociais e de saúde orientadas à esta faixa etária.

\section{Referências}

1. Diener E. Subjective well-being. Psychol Bull 1984; 95(3):542-575.

2. Enkvist A, Ekstrom H, Elmstahl S. What factors affect life satisfaction (LS) among the oldest-old? Arch Gerontol Geriatr 2012; 54(1):140-145.

3. Diener E, Suh EM, Lucas RE, Smith HL. Subjective well-being: three decades of progress. Psychol Bull 1999; 125(2):276-302.

4. Enkvist A, Ekstrom H, Elmstahl S. Life satisfaction (LS) and symptoms among the oldest-old: results from the longitudinal population study called Good Aging in Skane (GAS). Arch Gerontol Geriatr 2012; 54(1):146-150.

5. Borg C, Hallberg IR, Blomqvist K. Life satisfaction among older people with reduced self-care capacity: the relationship to social, health and financial aspects. J Clin Nurs 2006; 15(5):607-618.

6. George LK. Still happy after all these years: research frontiers on subjective well-being in later life. $J$ Gerontol B Psychol Sci Soc Sci 2010; 65B(3):331-339.

7. Di Nubila HBV. Uma introdução à CIF - Classificação Internacional de Funcionalidade, Incapacidade e Saúde. RBSO 2010; 35(121):122-123.

8. Garatachea N, Molinero O, Martinez-Garcia R, Jimenez-Jimenez R, Gonzalez-Gallego, Marquez S. Feelings of well being in elderly people: relationship to physical activity and physical function. Arch Gerontol Geriatr 2009; 48(3):306-312.

9. Marengoni A, Angleman S, Melis R, Mangialasche F, Karp A, Garmen A, Meinow B, Fratiglioni L. Aging with multimorbidity: A systematic review of the literature. Ageing Res Rev 2011; 10(4):430-439.

10. Lima-Costa MF, Barreto SM, Giatti L. Condições de saúde, capacidade funcional, uso de serviços de saúde e gastos com medicamentos da população idosa brasileira: um estudo descritivo baseado na Pesquisa Nacional por Amostra de Domicílios. Cad Saude Publica 2003; 19(3):735-743. 
11. Reuben DB, Laliberte L, Hiris J, Mor V. A hierarquical exercise scale to measure function at the advanced activities of daily living (AADL) level. $J$ Am Geriatr Soc 1990; 38(8):855-861.

12. Reuben DB, Wieland D, Rubenstein LZ. Functional status assessment of older persons: concepts and implications. Facts Res Gerontol 1993; 7:231-240.

13. Tsubaki A, Kubo M, Kobayashi R, Jigami H, Takahashi HE. Age-related changes in physical function in community-dwelling people aged 50-79 year. J Phys Ther Sci 2010; 22(1):23-27.

14. Scheibe S, Carstensen LL. Emotional aging: recent findings and future trends. J Gerontol B Psychol Sci Soc Sci 2010; 65B(2):135-144.

15. Neri AL. Dependência e autonomia. In: Guariento ME, Neri AL, organizadores. Assistência ambulatorial ao idoso. Campinas: Editora Alínea; 2010. p. 31-44.

16. Ozturk A, Simsek TT, Yumin ET, Sertel M, Yumin M. The relationship between physical, functional capacity and quality of life (QoL) among elderly people with a chronic disease. Arch Gerontol Geriatr 2011; 53(3):278-283

17. Lee CT, Yeh CJ, Lee MC, Lin HS, Chen VC, Hsieh $\mathrm{MH}$, Yen $\mathrm{CH}$, Lai TJ. Leisure activity, mobility limitation and stress as modifiable risk factors for depressive symptoms in the elderly: results of a national longitudinal study. Arch Gerontol Geriatr 2012; 54(2):221-229.

18. Cumming E, Henry WE. Growing old: The process of disengagement. New York: basic Books; 1961.

19. Neugarten BL, Havighurst RJ, Tobin SS. The measurement of life satisfaction. J Gerontol 1961; 16:131143.

20. Rowe J, Kahn R. Human aging: Usual and successful (physiological changes associated with aging). Science 1987; 237(4811):143-147.

21. World Health Organization (WHO). Active ageing: A policy framework. Geneva: WHO; 2002.

22. Neri AL, Yassuda MS, Fortes-Burgos ACG, Mantovani EP, Arbex FS, Torres SVS, Silva DD. FIBRA Campinas: fundamentos e metodologia de um estudo sobre fragilidade em idosos da comunidade. In: Neri AL, Guariento ME, organizadoras. Fragilidade, saúde e bem estar em idosos: dados do estudo FIBRA Campinas. Campinas: Ed. Alínea, 2011.

23. Instituto Brasileiro de Geografia e Estatística (IBGE). Contagem da população 2007. Resultados da publicação divulgada em 21/12/2007. [página na Internet]; 2008 [acessado 2008 jan 12]. Disponível em: HTTP:/ /www.ibge.gov.br/home/estatística/população/cc.

24. Fried LP, Tangen CM, Walston J, Newman AB, Hirsch C, Gottdiener J, Seeman T, Tracy R, Kop WJ, Burke G, McBurnie MA; Cardiovascular Health Study Collaborative Research Group. Frailty in older adults: evidence for a phenotype. J Gerontol Med Sci 2001; 56A:146-156

25. Brucki, SMD, Nitrini R, Caramelli P, Bertolucci PHF, Okamoto IH. Sugestões para o uso do Mini-Exame do Estado Mental no Brasil. Arq Neuropsiquiatr 2003; 61(3B):777-781.

26. Guralnik JM, Simonsick EM, Ferrucci L, Glynn RJ, Berkman LF, Blazer DG, Scherr PA, Wallace RB. A short physical performance battery assessing lower extremity function: association with self-reported disability and prediction of mortality and nursing home admissions. J Gerontol Med Sci 1994; 49(2):85-94.
27. Neri AL. Bienestar subjetivo em la vida adulta y em la vejez: hacia una psicologia positiva em America Latina. Rev. Latinoam Psicol. 2002; 34(1-2):55-74.

28. Lima-Costa MF, Firmo JOA, Uchoa E. The Bambuí Cohort Study of Aging: methodology and health profile of participants at baseline. Cad Saude Publica 2011; 27(3):327-335.

29. Bandeen-Roche K, Xue QL, Ferrucci L, Walston J, Guralnik JM, Chaves P, Zeger SL, Fried LP. Phenotype of frailty: characterization in the Women's Health and Aging Study. J Gerontol Med Sci 2006; 61A(3):262-266.

30. Breda JC. Prevalência de quatro critérios para avaliação da fragilidade em uma amostra de idosos residentes na comunidade: um estudo exploratório. [dissertação]. Campinas: Unicamp; 2007.

31. Camarano AA. Envelhecimento da população brasileira: uma contribuição demográfica. In: Freitas EV, Py L, Cançado FAX, Doll J, Gorzoni ML, organizadores. Tratado de Geriatria e Gerontologia. Rio de Janeiro: Guanabara Koogan; 2006. p. 88-105.

32. Lebrão ML, Laurenti, R. Saúde, bem-estar e envelhecimento: o estudo SABE no Município de São Paulo. Rev. Bras Epidemiol 2005; 8(2):127-141.

33. Louvison MCP, Lebrão ML, Duarte YAO, Santos JLF, Malik AM, Almeida, ES. Desigualdades no uso e acesso aos serviços de saúde entre idosos do município de São Paulo. Rev Saude Publica 2008; 42(4):733-740.

34. Neri AL. Feminização da velhice. In: Neri AL, organizadora. Idosos no Brasil: vivências, desafios e expectativas na terceira idade. São Paulo: Editora Fundação Perseu Abramo, edições SESC-SP; 2007. p. 47-64.

35. Oswald F, Wahl HW, Naumann D, Mollenkopf H, Hieber, A. The role of the home environment in middle and late adulthood. In: Wahl HW, Brenner $\mathrm{H}$, Mollenkopf H, Rothenbacher D, Rott C, organizadores. The many faces of health, competence and well-being in old age: integrating epidemiological, psychological and social perspectives. Heidelberg: Springer; 2006. p. 7-24.

36. Doimo LA, Derntl AM, Lago OC. O uso do tempo no cotidiano de mulheres idosas: um método indicador do estilo de vida de grupos populacionais. Cien Saude Colet 2008; 13(4):1133-1142.

37. Li YP, Lin SI, Chen CH. Gender differences in the relationship of social activity and quality of life in community-dwelling taiwanese elders. J Women Aging 2012; 23(4):305-320.

38. Buchman AS, Boyle PA, Wilson RS, Fleischman DA, Leurgans S, Bennett DA. Association between latelife social activity and motor decline in older adults. Arch Intern Med 2009; 169(12):1139-1146.

39. James BD, Boyle PA, Buchman AS, Bennett DA. Relation of late-life social activity with incident disability among community - dwelling older adults. $J$ Gerontol Med Sci 2011; 66A(4):467-473.

40. Smith J, Borchelt M, Maier H, Jopp D. Health and well-being in the young and oldest old. J Soc Issues 2002; 58(4):715-732.

Artigo apresentado em 18/10/2012

Aprovado em 27/11/2012

Versão final apresentada em 01/12/2012 